\title{
Assessment of Quality of Life Among Health Professionals During COVID-19: Review
}

\section{Usha Rani Kandula (D) \\ Addisu Dabi Wake}

Nursing Department, College of Health Sciences, Arsi University, Asella, Oromia Regional State, Ethiopia
Correspondence: Usha Rani Kandula College of Health Sciences, Arsi University, Oromia Region, P.O.Box-396, Asella, Ethiopia

Tel +25I-939052408

Email neha2010dec@gmail.com;

Id-usharani2020@arsiun.edu.et

\begin{abstract}
In 2019, coronavirus disease pandemic (COVID-19) influences the quality of life of health personnel who are on the front lines in dealing with COVID-19 patients. The purpose of this study is to assess the quality of life of health professionals during the COVID-19 epidemic. The novel coronavirus disease-19 (COVID-19) has spread across the globe with the direct causal viral agent of severe acute respiratory syndrome-corona virus-2 (SARS-CoV-2) and infected many people. All health professionals (HPs) such as physicians, nurses, and other allied health staff members are primary caregivers in hospitals and other health care settings, specifically under pandemic situations such as COVID-19. The emergence of the COVID-19 pandemic has caused numerous diversions in the preservation of the quality of life $(\mathrm{QoL})$ of health professionals by deviations from normal physical, mental, and social wellbeing aspects. HPs are the most vulnerable population to COVID-19 viral transmission while delivering emergency medical services to persons infected with the virus in various health care sectors, such as direct engagement in lifesaving management. In this perspective, some recent literature on QoL of health professionals was examined, uncovering that they frequently experience fear and anxiety due to viral transmissions in the place of work and probable cross-contamination among family members, tend to experience insomnia caused by sudden increased work pressure, struggle to balance professional and personal life, suffering from mental health disturbances such as depressive episodes, mood disturbances, and suicidal tendencies due to the unpredictability of the disease outbreak. The current review is looking to determine and address the degree of QoL maintained by specific types of health professionals during critical moments of COVID-19 pandemics. This study may assist health organization stakeholders in enhancing QoL among health professionals by introducing required provisions, measures, or initiatives for the welfare of health professionals, notably in resolving pandemic demands in all health organizations.
\end{abstract}

Keywords: assessment, quality of life, health professionals, COVID-19, SARS-CoV-2

\section{Introduction}

The severe acute respiratory syndrome-coronavirus-2 (SARS-COV-2) pandemic, designated coronavirus disease-19 (COVID-19), reportedly emerged in Wuhan, China, at the end of December 2019, then expanded all across the province, causing massive attention around the world. ${ }^{1-8}$ this virus can be actively spread from person to person by droplets emitted when coughing and sneezing. SARS-COV-2 infects the respiratory system, specifically the cells lining the alveoli, in humans. ${ }^{2,5,7,9,10}$ The first instance of COVID-19 was confirmed in Wuhan, Hubei Region, China. According to estimates, five SARS patients were treated first before the disease spread to all other countries. ${ }^{2,7,11,12}$ The World Health Organization declared the COVID-19 outbreak a global pandemic in March 2020.,13-15 
The risk factors are close contact, particularly living in the same house with a COVID-19 patient, and travel history to and from infected locations have been identified by the Centres for Disease Control and Prevention.,16 According to the World Health Organization (WHO), as of August 6, 2021, there were 200,840,180 confirmed positive COVID-19 cases, with a mortality rate of $4,265,903$, and vaccination status of $3,984,596,440 .{ }^{4}$ COVID-19 individual's clinical manifestations range from asymptomatic, moderate symptoms to pneumonia, severe pneumonia, ARDS, sepsis, and septic shock. ${ }^{2,5,16}$ As the front-line inpatient management, medical professionals or health care workers are among the populations under a significant chance of infection. ${ }^{2,10,12,17}$ There are several problems in the healthcare workplace and personal life, most of which are unforeseen during the epidemic of COVID-19. ${ }^{11,17-19}$

Quality of life is an individual's perspective of their place in life concerning the cultural background and belief systems in which they live, which are connected to aspirations, expectations, standards, and considerations. ${ }^{2,7,19}$ During the pandemic, HPs play a critical role in providing emergency medical services to all SARS-COV-2 virusinfected patients. ${ }^{2,14,20}$ In today's healthcare environment, indicators of work stress are a major public health problem. Certain work and personal related aspects may have an impact on HCWs' quality of life. ${ }^{1,19,26}$ The unprecedented conditions of persistent fear and anxiety may have a significant impact on the QoL of HCWs. ${ }^{3,19,27}$ The present COVID-19 epidemic has placed unprecedented strain on healthcare workers (HCWs) all across the world and impacted the nature of the work of the HPs. ${ }^{7,13,14}$ First and foremost, ensuring the safe and effective protection of HCWs from infection throughout the pandemic was essential not only to maintaining positive and healthy patient care but also to preventing outbreaks. ${ }^{3,10,12,19,20}$ During the SARS-COV-2 epidemic, health providers' quality of life might diminish. They are the front-line warriors in the struggle against the COVID-19 pandemic, and they are subjected to demanding work shifts and an increased and continuous risk of infection. ${ }^{2,17-19}$

Under great pressure, the HCWs would have to make life-saving judgments. These decisions included using limited resources appropriately and equally between many patients, how to manage their own physical and mental well-being necessities with those of their patients, and how to balance their duty obligations to patients with those to their family and friends, and how to continue providing medical care for all affected patients despite limited or insufficient resources. ${ }^{3,20,21}$ The WHO first addressed this issue, stating that the HPs should be safeguarded during pandemic problem periods by establishing safety measures to protect them from occupational illnesses. According to the literature, HPs are more vulnerable to the development of psychological illness during the onset of pandemic situations. $^{21-23}$ Depression and anxiety are thought to be triggered by the COVID-19 pandemic among health professionals. It may also cause restlessness, frustration, depressed mood, PTSD. Especially, people who have lost their autonomy, been separated from loved ones, or lost their professions are examples of this and it may be caused by fear of transmission and ambiguous sources of information shared on social networks. ${ }^{2,9,10,12}$

According to several research studies, HCWs who feared infection of their dear ones reported significant levels of stress, anxiety, and depression symptoms, which might have long-term psychological consequences. Comprehensive cross-sectional research of over 1000 Chinese HCWs found $71.5 \%$ distress, $50.4 \%$ depression, $44.6 \%$ anxiety, and $34.0 \%$ insomnia, correspondingly. The best information, based on a systematic review and metaanalyses of 13 research involving 33,062 participants, indicated that a considerable percentage of HCWs are reporting severe levels of anxiety, depression, and insomnia during the COVID-19 pandemic.

Anxiety and depression were prevalent in $23.2 \%$ and $22.8 \%$, respectively. It revealed that a large percentage of HCWs experienced mild symptoms of depression and anxiety, whereas moderate and severe symptoms were less prevalent. ${ }^{1,8,18,19,26}$ According to estimations, healthcare professionals may account for $20 \%$ of all diagnoses, carrying with them the anxiety and possibility of mortality from the viral infection, as well as intolerable psychological stress that can impact their quality of life. ${ }^{18,21}$

Some existing works on literature are reported that the average ratio of the hospital infected staff was $3.8 \%$, with maybe the cause of unprotected contacts with highly infected patients, with unknowingly infected patients, minimized personal protective equipment (PPE) while performing the emergency medical procedures in the health care settings. HPs are exposed to uncertain situations during the working hours, and there is a negative impact of falling on their personal life with unbalancing roles from the increased professional working demands. ${ }^{6,11,24-26}$ According to multiple studies, HPs are more concerned about a pandemic outbreak, and their family members' health concerns cause them to 
have severe psychological symptoms. HPs are well protected, with enough preventative and protective measures in place, while giving care to pandemic patients in any emergency circumstance. ${ }^{13,14,26}$

For the first time, taking care of patients was united with HCWs' concern for their patient's health, uncertainties, emotional disturbances, and stigmas. HCWs expressed contradictory thoughts and difficulties in balancing their professional roles and their household responsibilities. They discussed the difficulties of balancing duty and a feeling of guilt. ${ }^{1,8,14,21,22}$ Work-related concerns, along with psychological quests, ethical-moral dilemmas, and patient expectations, can put a strain on the professional's emotional condition. Numerous research found that HCWs who feared contagion were more likely to be reluctant to work or to consider quitting. $3,21,22$

\section{Assessment of Quality of Life Among Health Professionals}

During the COVID-19 pandemic, several research studies on QoL among HPs were published by scholars from various countries. The researcher gathered information from the Google Scholar database by using the search terms Quality of life "AND" health professionals "AND" COVID-19 in July 2021. The collected data are summarised and presented in the form of a literature review to evaluate the QoL status of health professionals during pandemic scenarios. Each review literature includes the research title, multiple tools used to measure QoL among health professionals, and research outcomes, and the data simplified in the tabular format consist of Author name, Title of the study, Study design, Study population, Sampling method/technique, Sample size, Data collection period, Year, Country, Method/Instruments used for QoL Assessment among HPs, Findings of the study (refer Table 1).

A cross-sectional study report from Indonesia on the quality of life among health workers (HW's) during the COVID-19 epidemic was discovered. The World Health Organization Quality Of Life-BREF (WHOQOL-BREF) instrument has been used to assess the HWs' Quality of Life (QoL). It has four categories of quality of life: physical health, psychological health, social relationships, and the environment, which are used as a method for assessing QoL, which included 26 questionnaire items. The study's findings were as follows: the Physical Health Domain average score $(63.18 \pm 10.62)$ and Psychological Health Domain average score $(60.33 \pm 15.44)$ were interpreted with Good QoL, whereas Social Relation Health Domain average score $(51.57 \pm 17.61)$ and Environmental Health Domain average score $(57.28 \pm 12.48)$ were interpreted with Moderate QoL among HWs. The study conclusions are all health professionals who participated in the study indicated good physical and psychological health with moderate social relationship quality and environmental health. $^{2}$

Cross-sectional research on health anxiety related to COVID-19 virus infection and its association to quality among health care professionals was reported from Egypt. The quality of life (QoL) of health care workers was investigated using the Short Health Anxiety Inventory (SHAI), the Symptom Check List-90-Revised (SCL-90-R), and the World Health Organization Quality of Life - BREF (WHOQOLBREF). According to the study's findings, $28 \%$ of HCWs experienced health worries about the COVID-19 virus. Health anxiety in response to the COVID-19 virus was inversely associated with all domains of QoL in HCWs. Quality of life (QoL) domains Mean and SD among physicians $(n=74)$ were the Physical health (55.2 \pm 14.5$)$, Psychological (58.7 \pm 17.3 ), Social relationship (60.8 \pm 20.2 ), Environmental (49.6 \pm 16.8 ), whereas forOther HCWs $(\mathrm{n}=144)$ were Physical health (49.2 \pm 15.7$)$, Psychological (56.1 \pm 14.3$)$, Social relationship (64.0 \pm 19.2$)$, Environmental (49.2 \pm 14.1$)^{3}{ }^{3}$

A cross-sectional study on Professional Quality of Life (QoL) and Mental Health Outcomes among SARS-COV-2 (COVID-19)-Affected Health Care Workers (HCWs) was published from Italy, comprising $265 \mathrm{HCWs}$. The Professional Quality of Life-5 (ProQOL-5), the NineItem Patient Health Questionnaire (PHQ-9), and the Seven-Item Generalized Anxiety Disorder scale (GAD-7) were used to assess compassion satisfaction (CS), burnout, secondary traumatization (ST), and depression and anxiety symptoms in HCWs, respectively. The whole sample, as well as the study's highlights, fulfillment of mean \pm SD of compassion satisfaction $(38.2 \pm 7.0)$, burnout $(19.8 \pm 5.0)$, secondary traumatic stress $(18.0 \pm 5.6)$. The study recognized that being in the front line and working in an ICU demonstrated to be a potential risk factor for anxiety but not for depressive symptoms. ${ }^{1}$

The effect of stress, anxiety, and burnout levels on the quality of life of healthcare personnel caring with COVID19 patients was investigated in a cross-sectional research study conducted in Turkey among 240 health care employees. The study employed the Perceived Stress Scale (PSS), Spielberger State-Trait Anxiety Inventory, Maslach Burnout Inventory, and Quality of Life (QoL) Scale. The study's main findings were that the stress, anxiety, and 
Table I Quality of Life Among Health Professionals During COVID-19

\begin{tabular}{|c|c|c|c|c|c|c|c|c|c|c|c|}
\hline $\begin{array}{l}\text { SI. } \\
\text { No }\end{array}$ & $\begin{array}{l}\text { Author } \\
\text { Name }\end{array}$ & Title of the Study & $\begin{array}{l}\text { Type of } \\
\text { Study } \\
\text { Design }\end{array}$ & Study Population & $\begin{array}{l}\text { Sampling } \\
\text { Method }\end{array}$ & $\begin{array}{l}\text { Study } \\
\text { Sample Size }\end{array}$ & $\begin{array}{l}\text { Data } \\
\text { Collection } \\
\text { Period }\end{array}$ & Year & Country & $\begin{array}{l}\text { Method/Instrument Used for } \\
\text { QoL Assessment }\end{array}$ & Findings of the Study \\
\hline 1 & Hadning $^{2}$ & $\begin{array}{l}\text { An analysis of health } \\
\text { workers' quality of life in } \\
\text { Indonesia during } \\
\text { COVID-19 pandemic }\end{array}$ & $\begin{array}{l}\text { Observation } \\
\text { with cross- } \\
\text { sectional } \\
\text { technique }\end{array}$ & Health workers & $\begin{array}{l}\text { Non- } \\
\text { probability } \\
\text { sampling } \\
\text { methods }\end{array}$ & $\begin{array}{l}\text { Among } 184 \\
\text { (114 women, } \\
70 \text { men) }\end{array}$ & $\begin{array}{l}\text { From I June } \\
\text { to August } \\
2020 \text { (I } \\
\text { month) }\end{array}$ & 2020 & Indonesia & $\begin{array}{l}\text { WHOQOL-BREF } \\
\text { World Health Organization QoL } \\
\text { assessment tool }\end{array}$ & $\begin{array}{l}\text { Respondents' Average Quality of } \\
\text { Life was determined as the } \\
\text { following: } \\
\text {-Physical Health Domain score - } \\
63,18 \pm 10,62 \text {. } \\
\text {-Psychological Health Domain } \\
\text { score - } 60,33 \pm 15,44 \text {. } \\
\text {-Social Relation Health Domain } \\
\text { score - } 51,57 \pm 17,61 \text {. } \\
\text {-Environmental Health Domain } \\
\text { score - } 57,28 \pm 12,48 \text {. }\end{array}$ \\
\hline 2 & Abdelghani ${ }^{3}$ & $\begin{array}{l}\text { Health anxiety to } \\
\text { COVID-19 virus } \\
\text { infection and its } \\
\text { relationship to quality of } \\
\text { life in a sample of health } \\
\text { care workers in Egypt: a } \\
\text { cross-sectional study }\end{array}$ & $\begin{array}{l}\text { Cross- } \\
\text { sectional } \\
\text { study }\end{array}$ & $\begin{array}{l}\text { Health care } \\
\text { workers }\end{array}$ & $\begin{array}{l}\text { Systematic } \\
\text { random } \\
\text { sampling } \\
\text { method }\end{array}$ & 218 & $\begin{array}{l}\text { From } 30 \\
\text { June to } 16 \\
\text { July }\end{array}$ & 2020 & Egypt & $\begin{array}{l}\text { Short Health Anxiety Inventory } \\
\text { (SHAI), } \\
\text { Symptom Check List-90-Revised } \\
\text { (SCL-90-R), } \\
\text { World Health Organization } \\
\text { Quality of Life - BREF } \\
\text { (WHOQOL-BREF). }\end{array}$ & $\begin{array}{l}\text { Quality of life (QOL) domains } \\
\text { Mean and SD among physicians } \\
(\mathrm{n}=74) \text {, was Physical health - } 55.2 \\
\text { (14.5), Psychological- } 58.7(17.3) \text {, } \\
\text { Social relationship- } 60.8(20.2), \\
\text { Environmental- } 49.6(16.8) \text {, } \\
\text { Whereas Other HCWs }(\mathrm{n}=144) \\
\text { was Physical health- } 49.2 \text { (15.7), } \\
\text { Psychological- } 56.1 \text { (14.3), Social } \\
\text { relationship- } 64.0(19.2) \text {, } \\
\text { Environmental- } 49.2(14.1) .\end{array}$ \\
\hline 3 & Buselli' & $\begin{array}{l}\text { Professional quality of } \\
\text { life and mental health } \\
\text { outcomes among health } \\
\text { care workers exposed } \\
\text { to SARS-CoV-2 } \\
\text { (COVID-19) }\end{array}$ & $\begin{array}{l}\text { Cross- } \\
\text { sectional } \\
\text { study }\end{array}$ & $\begin{array}{l}\text { Health care } \\
\text { workers }\end{array}$ & Online survey & $\begin{array}{l}265 \\
84 \text { Men } \\
181 \text { Women }\end{array}$ & $\begin{array}{l}\text { I April and } \\
\text { I May }\end{array}$ & 2020 & Italy & $\begin{array}{l}\text { Professional Quality of Life-5 } \\
\text { (ProQOL-5), the Nine-Item } \\
\text { Patient Health Questionnaire } \\
\text { (PHQ-9), and the Seven-Item } \\
\text { Generalized Anxiety Disorder } \\
\text { scale (GAD-7) }\end{array}$ & $\begin{array}{l}\text { The overall sample, }(\mathrm{N}=265) \\
\text { Compassion Satisfaction (Mean } \pm \\
\text { SD) } 38.2 \pm 7.0 \text {, Burnout (Mean } \pm \\
\text { SD) } 19.8 \pm 5.0 \text {, Secondary } \\
\text { Traumatic Stress (Mean } \pm \text { SD) } \\
18.0 \pm 5.6 \text {, PHQ-9 (Mean } \pm \text { SD) } \\
4.5 \pm 6.4, \text { GAD-7 (Mean } \pm \text { SD) } \\
4.2 \pm 4.6 \text {. }\end{array}$ \\
\hline
\end{tabular}




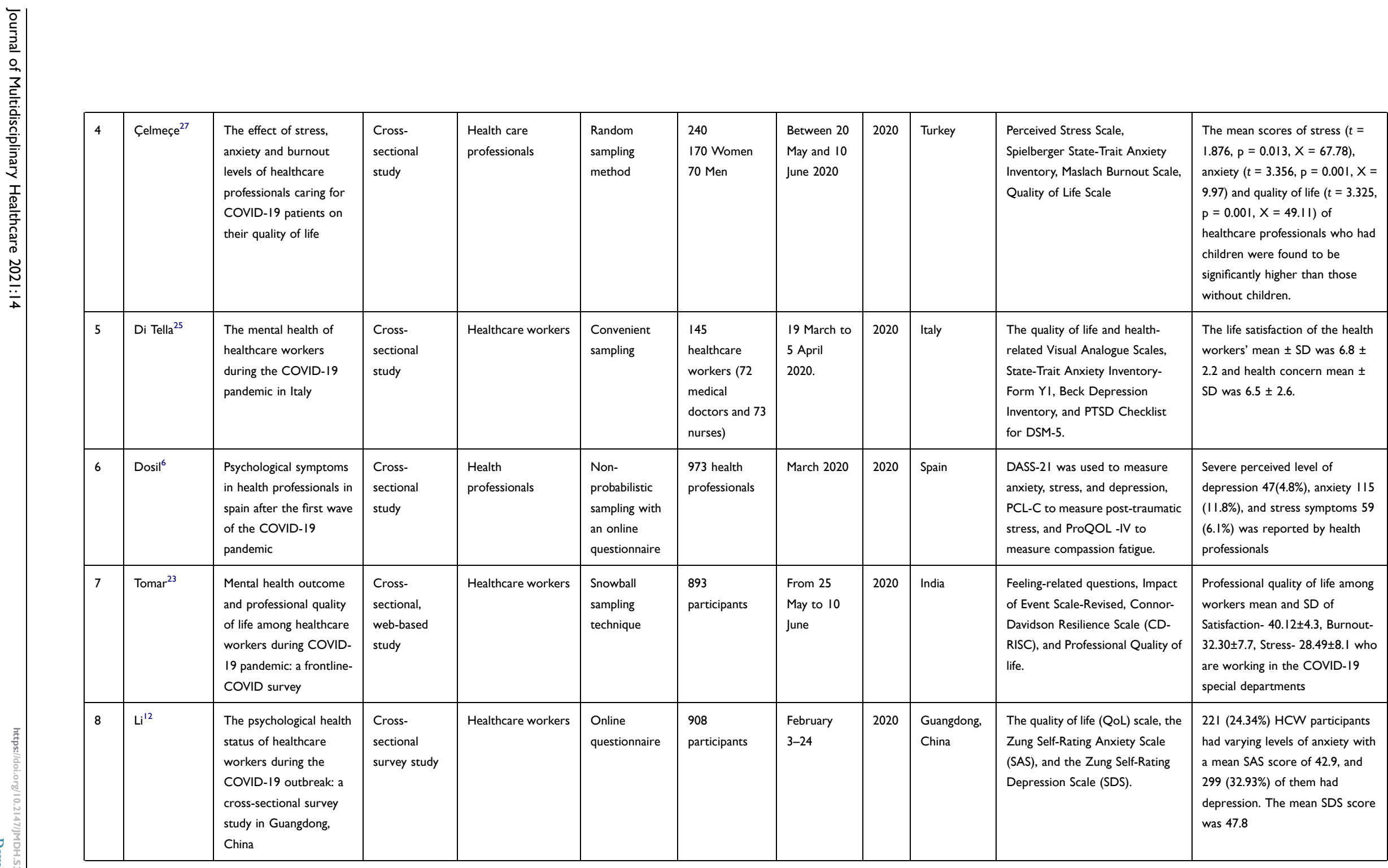

(Continued) 
Table I (Continued).

\begin{tabular}{|c|c|c|c|c|c|c|c|c|c|c|c|}
\hline $\begin{array}{l}\text { SI. } \\
\text { No }\end{array}$ & $\begin{array}{l}\text { Author } \\
\text { Name }\end{array}$ & Title of the Study & $\begin{array}{l}\text { Type of } \\
\text { Study } \\
\text { Design }\end{array}$ & Study Population & $\begin{array}{l}\text { Sampling } \\
\text { Method }\end{array}$ & $\begin{array}{l}\text { Study } \\
\text { Sample Size }\end{array}$ & $\begin{array}{l}\text { Data } \\
\text { Collection } \\
\text { Period }\end{array}$ & Year & Country & $\begin{array}{l}\text { Method/Instrument Used for } \\
\text { QoL Assessment }\end{array}$ & Findings of the Study \\
\hline 9 & Manh Than ${ }^{20}$ & $\begin{array}{l}\text { Mental health and } \\
\text { health-related quality-of- } \\
\text { life outcomes among } \\
\text { frontline health workers } \\
\text { during the peak of } \\
\text { COVID-19 outbreak in } \\
\text { Vietnam: a cross- } \\
\text { sectional study }\end{array}$ & $\begin{array}{l}\text { Cross- } \\
\text { sectional } \\
\text { research } \\
\text { study }\end{array}$ & $\begin{array}{l}\text { Frontline health } \\
\text { workers }\end{array}$ & $\begin{array}{l}\text { Convenience } \\
\text { sampling }\end{array}$ & $\begin{array}{l}173 \text { Health } \\
\text { workers }\end{array}$ & $\begin{array}{l}\text { March to } \\
\text { April }\end{array}$ & 2020 & Vietnam & $\begin{array}{l}\text { Depression, Anxiety, and Stress } \\
\text { Scale - } 2 \text { I Items (DASS-2I), } \\
\text { Impact of Event Scale-Revised } \\
\text { (IES-R), and the Insomnia Severity } \\
\text { Index (ISI), EQ-5D-5L. }\end{array}$ & $\begin{array}{l}\text { Mental Health and HRQoL } \\
\text { Outcomes of HCWs during the } \\
\text { COVID-19 Outbreak in the } \\
\text { Designated Hospital ( } N=106) \text {, as } \\
\text { per the EQ-5D-5L profile, the } \\
\text { components are responded as } \\
\text { Mobility, } n(\%)-34(32.1 \%) \text {, Self- } \\
\text { care, } n(\%)-9(8.5 \%), \text { Usual } \\
\text { activities, } n(\%)-22(20.8 \%) \text {, Pain/ } \\
\text { Discomfort, } n(\%)-29(27.4 \%) \text {, } \\
\text { Anxiety/Depression, } n(\%)-84 \\
(79.3 \%) \text {. }\end{array}$ \\
\hline 10 & $\begin{array}{l}\text { Ortega- } \\
\text { Galán }\end{array}$ & $\begin{array}{l}\text { Professional quality of } \\
\text { life and perceived stress } \\
\text { in health professionals } \\
\text { before COVID-19 in } \\
\text { Spain: primary and } \\
\text { hospital care }\end{array}$ & $\begin{array}{l}\text { Cross- } \\
\text { sectional } \\
\text { observational } \\
\text { study }\end{array}$ & $\begin{array}{l}\text { Health } \\
\text { professionals }\end{array}$ & $\begin{array}{l}\text { Convenient } \\
\text { with Online } \\
\text { questionnaire }\end{array}$ & 537 & $\begin{array}{l}30 \text { March } \\
\text { until } 16 \\
\text { April }\end{array}$ & 2020 & Spain & $\begin{array}{l}\text { Professional Quality of Life (QoL) } \\
\text { and Perceived Stress (PSS-14) }\end{array}$ & $\begin{array}{l}\text { Professional quality of life and } \\
\text { perceived stress in-hospital care } \\
\text { related to, the highest } \\
\text { compassion satisfaction was } 125 \\
(42.5 \%) \text {, compassion fatigue was } \\
189(64.3 \%) \text { and burnout was } 108 \\
(36.7 \%)\end{array}$ \\
\hline 11 & Stojanov ${ }^{22}$ & $\begin{array}{l}\text { Quality of sleep and } \\
\text { health-related quality of } \\
\text { life among health care } \\
\text { professionals treating } \\
\text { patients with } \\
\text { coronavirus disease-19 }\end{array}$ & $\begin{array}{l}\text { Cross- } \\
\text { sectional, } \\
\text { web-based } \\
\text { study }\end{array}$ & $\begin{array}{l}\text { Health care } \\
\text { professionals }\end{array}$ & $\begin{array}{l}\text { Random } \\
\text { sampling }\end{array}$ & $\begin{array}{l}201 \\
\text { participants }\end{array}$ & March 20 & 2020 & Serbia & $\begin{array}{l}\text { 7-item Generalized Anxiety } \\
\text { Disorder (GAD-7) Scale, Zung } \\
\text { Self-rating Depression Scale, 36- } \\
\text { item Health Survey of the Medical } \\
\text { Outcomes Study Short Form } \\
\text { (SF36), Pittsburgh Sleep Quality } \\
\text { Index (PSQI). }\end{array}$ & $\begin{array}{l}\text { In group I, the assessment as } \\
\text { Group I ( } N=118) \text {, the scores } \\
\text { was PSQI global score }-8.3 \pm 4.5 \text {, } \\
\text { SF36 total score- } 80.06 \pm 24.69 \text {, } \\
\text { GAD-7 - } 13.26 \pm 5.32 \text {, SDS- } \\
53.14 \pm 11.4 I \text {. } \\
\text { Group II (N = 83), PSQI global } \\
\text { score-5.2 } \pm 3.7 \text {, SF36 total score- } \\
86.14 \pm 25.13 \text {, GAD-7-8.25 } \pm \\
5.61 \text {, SDS-49.39 } \pm 10.61 \text {. }\end{array}$ \\
\hline 12 & Suryavanshi ${ }^{29}$ & $\begin{array}{l}\text { Mental health and quality } \\
\text { of life among healthcare } \\
\text { professionals during the } \\
\text { COVID-19 pandemic in } \\
\text { India. }\end{array}$ & $\begin{array}{l}\text { Cross- } \\
\text { sectional } \\
\text { study }\end{array}$ & $\begin{array}{l}\text { Healthcare } \\
\text { professionals }\end{array}$ & $\begin{array}{l}\text { Snowball } \\
\text { sampling, }\end{array}$ & 197 & $\begin{array}{l}5 \text { May } 2020 \\
\text { to } 16 \text { May } \\
2020\end{array}$ & 2020 & India & $\begin{array}{l}\text { Patient Health Questionnaire } \\
\text { (PHQ-9), Generalized Anxiety } \\
\text { Disorder (GAD-7) questionnaire, } \\
\text { quality of life (QoL-I) visual } \\
\text { analogue scale. }\end{array}$ & $\begin{array}{l}\text { The low quality of life reported in } \\
\text { the study was n= } 89(45 \%) \text { with } \\
\text { moderate-to-severe depression } \\
\text { was } 32(73 \%) \text {, moderate-to- } \\
\text { severe anxiety was } 39(70 \%) \text {, and } \\
\text { moderate-to-severe depression } \\
\text { and anxiety combined was } 24 \\
(73 \%)\end{array}$ \\
\hline
\end{tabular}




\begin{tabular}{|c|c|c|c|c|c|c|c|c|c|c|c|}
\hline 13 & $\operatorname{Toh}^{10}$ & $\begin{array}{l}\text { Mental health status of } \\
\text { healthcare versus other } \\
\text { essential workers in } \\
\text { Australia amidst the } \\
\text { COVID-19 pandemic: } \\
\text { initial results from the } \\
\text { collate project }\end{array}$ & $\begin{array}{l}\text { Cross- } \\
\text { sectional } \\
\text { study }\end{array}$ & $\begin{array}{l}\text { Healthcare versus } \\
\text { other essential } \\
\text { workers }\end{array}$ & $\begin{array}{l}\text { Snowball } \\
\text { sampling }\end{array}$ & $\begin{array}{l}\text { Healthcare } \\
\text { workers } \\
\text { (HCW; } \\
\mathrm{n}=905) \text {, other } \\
\text { essential } \\
\text { workers } \\
\text { (OEW; } \\
\mathrm{n}=810 \text { ), and } \\
\text { the general } \\
\text { population } \\
\text { (GNP; } \\
\mathrm{n}=3443) \text {. }\end{array}$ & | April 2021 & 2021 & Australia & $\begin{array}{l}\text { Depression Anxiety Stress Scales } \\
\text { (DASS-2I, Positive and Negative } \\
\text { Affect Schedule (PANAS; } \\
\text { European Health Interview } \\
\text { Surveys - Quality of Life } \\
\text { (EUROHIS-QoL). }\end{array}$ & $\begin{array}{l}\text { Marginal mean } \pm \text { standard error } \\
\text { of healthcare worker (HCW; } \\
\mathrm{n}=879-905 \text { ) and Quality of life } \\
\text { (EUROHIS-QoL, } \alpha=0.869 \text { ) 777.I } \\
\pm 17.9 \text {. }\end{array}$ \\
\hline 14 & $\begin{array}{l}\text { Turcu- } \\
\text { Stiolica }{ }^{14}\end{array}$ & $\begin{array}{l}\text { Influence of COVID-19 } \\
\text { on health-related quality } \\
\text { of life and the perception } \\
\text { of being vaccinated to } \\
\text { prevent COVID-19: an } \\
\text { approach for community } \\
\text { pharmacists from } \\
\text { Romania and Bulgaria }\end{array}$ & $\begin{array}{l}\text { Cross- } \\
\text { sectional } \\
\text { study }\end{array}$ & $\begin{array}{l}\text { Community } \\
\text { pharmacists }\end{array}$ & $\begin{array}{l}\text { Convenience } \\
\text { sampling }\end{array}$ & 176 & $\begin{array}{l}15 \text { July to } 15 \\
\text { August } 2020\end{array}$ & 2020 & $\begin{array}{l}\text { Romania } \\
\text { and Bulgaria }\end{array}$ & $\begin{array}{l}\text { 15D instrument was used for } \\
\text { quality-of-life assessment in the } \\
\text { study. }\end{array}$ & $\begin{array}{l}\text { The total I5D score was in } \\
\text { Romania }(n=24 I)-0.956 \pm \\
0.05 I \text { and Bugaria }(n=154)- \\
0.936 \pm 0.063 .\end{array}$ \\
\hline 15 & Ungureanu $^{30}$ & $\begin{array}{l}\text { Impact of the COVID-19 } \\
\text { pandemic on health- } \\
\text { related quality of life, } \\
\text { anxiety, and training } \\
\text { among young } \\
\text { gastroenterologists in } \\
\text { Romania }\end{array}$ & $\begin{array}{l}\text { Cross- } \\
\text { sectional } \\
\text { study }\end{array}$ & Gastroenterologists & $\begin{array}{l}\text { Convenient } \\
\text { sampling }\end{array}$ & 174 & $\begin{array}{l}21 \text { April } 21 \\
\text { to } 9 \text { May } \\
2020\end{array}$ & 2020 & Romania & $\begin{array}{l}\text { The validated instruments are } \\
\text { 15D (for assessing the health- } \\
\text { related quality of life) and Endler } \\
\text { multidimensional anxiety scales } \\
\text { (EMAS-for assessing anxiety). }\end{array}$ & $\begin{array}{l}\text { The identified HRQoL among the } \\
\text { Gastroenterology fellow group }(\mathrm{n} \\
=64) \text { was } 0.966(0.055) \text { and } \\
\text { Young specialist group }(\mathrm{n}=32) \\
\text { was } 0.966(0.036) \text {. }\end{array}$ \\
\hline 16 & Vafaeil' & $\begin{array}{l}\text { Obstetrics healthcare } \\
\text { providers' mental health } \\
\text { and quality of life during } \\
\text { COVID-19 pandemic: } \\
\text { multicenter study from } \\
\text { eight cities in Iran }\end{array}$ & $\begin{array}{l}\text { Cross- } \\
\text { sectional } \\
\text { multicenter } \\
\text { study }\end{array}$ & $\begin{array}{l}\text { Obstetrics } \\
\text { healthcare } \\
\text { providers }\end{array}$ & $\begin{array}{l}\text { Snowball } \\
\text { sampling } \\
\text { through social } \\
\text { networking }\end{array}$ & 599 & $\begin{array}{l}\text { From } 9 \text { to } \\
16 \text { March }\end{array}$ & 2020 & Iran & $\begin{array}{l}\text { The Patient Health Questionaire- } \\
9 \text { (PHQ-9), Multidimensional } \\
\text { Scale of Perceived Social Support } \\
\text { (MSPSS), and Short Form-36 (SF- } \\
\text { 36). }\end{array}$ & $\begin{array}{l}\text { The Quality of life under Physical } \\
\text { aspect components of Physical } \\
\text { functioning was } 90 \text { [80-100], } \\
\text { Limitations due to physical health } \\
\text { was } 50 \text { [25-100], Pain was } 80 \\
\text { [55-100], General health was } 75 \\
\text { [55-90]. }\end{array}$ \\
\hline
\end{tabular}

(Continued) 
Table I (Continued).

\begin{tabular}{|c|c|c|c|c|c|c|c|c|c|c|c|}
\hline $\begin{array}{l}\text { SI. } \\
\text { No }\end{array}$ & $\begin{array}{l}\text { Author } \\
\text { Name }\end{array}$ & Title of the Study & $\begin{array}{l}\text { Type of } \\
\text { Study } \\
\text { Design }\end{array}$ & Study Population & $\begin{array}{l}\text { Sampling } \\
\text { Method }\end{array}$ & $\begin{array}{l}\text { Study } \\
\text { Sample Size }\end{array}$ & $\begin{array}{l}\text { Data } \\
\text { Collection } \\
\text { Period }\end{array}$ & Year & Country & $\begin{array}{l}\text { Method/Instrument Used for } \\
\text { QoL Assessment }\end{array}$ & Findings of the Study \\
\hline 17 & $X i e^{31}$ & $\begin{array}{l}\text { Workplace violence and } \\
\text { its association with } \\
\text { quality of life among } \\
\text { mental health } \\
\text { professionals in China } \\
\text { during the COVID-19 } \\
\text { pandemic }\end{array}$ & $\begin{array}{l}\text { Cross- } \\
\text { sectional } \\
\text { study }\end{array}$ & $\begin{array}{l}\text { Mental health } \\
\text { professionals }\end{array}$ & $\begin{array}{l}\text { Convenient } \\
\text { sampling with } \\
\text { weChat-based } \\
\text { Questionnaire }\end{array}$ & $\begin{array}{l}10,516 \\
\text { participants }\end{array}$ & $\begin{array}{l}\text { Between } 15 \\
\text { and } 20 \\
\text { March } 2020\end{array}$ & 2020 & China & $\begin{array}{l}\text { 9-item workplace violence scale, } \\
\text { 7-item Generalized Anxiety } \\
\text { Disorder Chinese version (GAD- } \\
\text { 7, QOL was evaluated using the } \\
\text { first two items on the overall } \\
\text { QOL derived from the World } \\
\text { Health Organization Quality of } \\
\text { Life Questionnaire - Brief Version } \\
\text { (WHOQOL-BREF). }\end{array}$ & $\begin{array}{l}\text { The overall, Quality of Life (QoL) } \\
\text { of mental health professionals } \\
\text { was 6.6(1.6\%), whereas the non- } \\
\text { WPV group ( } \mathrm{N}=8568 \text { ) QoL was } \\
6.8(1.5 \%) \text { and WPV group ( } \mathrm{N}= \\
\text { 1948) QoL was } 5.9(1.5 \%)\end{array}$ \\
\hline 18 & Young ${ }^{21}$ & $\begin{array}{l}\text { Health care workers' } \\
\text { mental health and quality } \\
\text { of life during COVID-19: } \\
\text { results from a mid- } \\
\text { pandemic, national } \\
\text { survey }\end{array}$ & $\begin{array}{l}\text { Cross- } \\
\text { sectional } \\
\text { study }\end{array}$ & $\begin{array}{l}\text { Health care } \\
\text { workers }\end{array}$ & $\begin{array}{l}\text { Convenient } \\
\text { sampling with } \\
\text { social media } \\
\text { support }\end{array}$ & $\begin{array}{l}1685 \\
\text { participants }\end{array}$ & $\begin{array}{l}\text { From I to } \\
28 \text { April }\end{array}$ & 2020 & U.S & $\begin{array}{l}\text { Patient Health Questionnaire-9, } \\
\text { General Anxiety Disorder-7, } \\
\text { Primary Care Posttraumatic } \\
\text { Stress Disorder Screen, and } \\
\text { Alcohol Use Disorders } \\
\text { Identification Test-C. }\end{array}$ & $\begin{array}{l}\text { Of I } 685 \text { participants, } 31 \% \text { ( } 404 \text { of } \\
\text { I3II) endorsed mild anxiety, and } \\
33 \% \text { ( } 427 \text { of I3II) clinically } \\
\text { meaningful anxiety; } 29 \% \text { ( } 393 \text { of } \\
\text { I34I) reported mild depressive } \\
\text { symptoms, and I7\% (233 of I34I) } \\
\text { moderate-to-severe depressive } \\
\text { symptoms; } 5 \% \text { ( } 64 \text { of I326) } \\
\text { endorsed suicidal ideation; and } \\
\text { I4\% (I84 of I300) screened } \\
\text { positive for posttraumatic stress } \\
\text { disorder. }\end{array}$ \\
\hline 19 & Zhang $^{32}$ & $\begin{array}{l}\text { Impact of the COVID-19 } \\
\text { pandemic on mental } \\
\text { health and quality of life } \\
\text { among local residents in } \\
\text { Liaoning Province, } \\
\text { China: a cross-sectional } \\
\text { study }\end{array}$ & $\begin{array}{l}\text { Cross- } \\
\text { sectional } \\
\text { study }\end{array}$ & $\begin{array}{l}\text { Local Chinese } \\
\text { residents }\end{array}$ & $\begin{array}{l}\text { Convenience } \\
\text { and snowball } \\
\text { sampling } \\
\text { methods }\end{array}$ & $\begin{array}{l}263 \\
\text { participants } \\
\text { 106-M } \\
\text { 157-F }\end{array}$ & $\begin{array}{l}\text { Between } \\
\text { January and } \\
\text { February }\end{array}$ & 2020 & $\begin{array}{l}\text { Liaoning } \\
\text { Province, } \\
\text { China }\end{array}$ & $\begin{array}{l}\text { The study instruments are the } \\
\text { Impact of Event Scale (IES), } \\
\text { Indicators of negative mental } \\
\text { health impacts, social and family } \\
\text { support, and mental health- } \\
\text { related lifestyle changes. }\end{array}$ & $\begin{array}{l}\text { The mean IES score in the } \\
\text { participants was } 13.6 \pm 7.7 \text {, } \\
\text { reflecting a mild stressful impact. } \\
\text { Only } 7.6 \% \text { of the participants had } \\
\text { an IES score } \geq 26 \text {. }\end{array}$ \\
\hline 20 & Zhang $^{33}$ & $\begin{array}{l}\text { Depression and its } \\
\text { relationship with quality } \\
\text { of life in frontline } \\
\text { psychiatric clinicians } \\
\text { during the COVID-19 } \\
\text { pandemic in China: a } \\
\text { national survey }\end{array}$ & $\begin{array}{l}\text { Cross- } \\
\text { sectional } \\
\text { study }\end{array}$ & $\begin{array}{l}\text { Frontline } \\
\text { psychiatric } \\
\text { clinicians }\end{array}$ & $\begin{array}{l}\text { Convenience } \\
\text { sampling }\end{array}$ & 10,516 & $\begin{array}{l}\text { From } 15 \text { to } \\
20 \text { March } \\
2020\end{array}$ & 2020 & China & $\begin{array}{l}\text { Depression and QOL were } \\
\text { assessed using the Patient Health } \\
\text { Questionnaire nine items (PHQ- } \\
\text { 9) and the World Health } \\
\text { Organization Quality of Life } \\
\text { Questionnaire-brief version } \\
\text { (WHOQOL-BREF). }\end{array}$ & $\begin{array}{l}\text { The overall (Quality of Life) QoL } \\
\text { of the study participants was } 6.64 \\
(1.60 \%) \text {, whereas the QoL of No } \\
\text { depression participants was } \\
(\mathrm{N}=7517)-7.12(1.42 \%) \text { and with } \\
\text { depression symptoms of } \\
\text { participants was }(\mathrm{N}=2999)-5.46 \\
(1.39 \%)\end{array}$ \\
\hline
\end{tabular}




\begin{tabular}{|c|c|c|c|c|c|c|c|c|c|c|c|}
\hline 21 & $\begin{array}{l}\text { Herrero San } \\
\text { Martin'19 }\end{array}$ & $\begin{array}{l}\text { Sleep characteristics in } \\
\text { health workers exposed } \\
\text { to the COVID-19 } \\
\text { pandemic }\end{array}$ & $\begin{array}{l}\text { Cross- } \\
\text { sectional } \\
\text { study }\end{array}$ & Health workers & $\begin{array}{l}\text { A stratified } \\
\text { sampling } \\
\text { technique with } \\
\text { subsequent } \\
\text { simple } \\
\text { randomization. }\end{array}$ & 170 & $\begin{array}{l}\text { From I } \\
\text { March to } 30 \\
\text { April } 2020\end{array}$ & 2020 & Spain & $\begin{array}{l}\text { Epworth Sleepiness Scale (ESS), } \\
\text { Pittsburgh Sleep Quality Index } \\
\text { (PSQI), Insomnia Severity Index } \\
\text { (ISI), and I7-items Hamilton } \\
\text { Rating Scale (HRS). }\end{array}$ & $\begin{array}{l}\text { Self-reported insomnia, } \\
\text { nightmares, sleepwalking, sleep } \\
\text { terrors are more among the } \\
\text { health workers with worse } \\
\text { quality of sleep disturbances. The } \\
\text { detected Insomnia Severity Index } \\
\text { (ISI) total score (mean } \pm \text { SD) } \\
\text { among health workers was } 7.83 \pm \\
5.29 \text { at p-value of } 0.05 .\end{array}$ \\
\hline 22 & Liu $^{9}$ & $\begin{array}{l}\text { Mental health status of } \\
\text { healthcare workers in } \\
\text { China for COVID-19 } \\
\text { eEpidemic }\end{array}$ & $\begin{array}{l}\text { Cross- } \\
\text { sectional } \\
\text { study }\end{array}$ & Healthcare workers & $\begin{array}{l}\text { Convenience, } \\
\text { snowball, } \\
\text { Random } \\
\text { sampling. }\end{array}$ & 1570 & $\begin{array}{l}\text { From } 29 \\
\text { January to } 3 \\
\text { February } \\
2020\end{array}$ & 2020 & China & $\begin{array}{l}\text { SCL-90 scale and a } \\
\text { sociodemographic questionnaire }\end{array}$ & $\begin{array}{l}52 \% \text { aspect of life most strongly } \\
\text { affected during the epidemic was } \\
\text { economic problems, and } \\
\text { interpersonal communication } \\
\text { problems, was } 46.6 \% \text {, followed by } \\
\text { mental health issues was } 46.4 \% \text {; } \\
\text { learn or work was } 45.0 \% \text {; body } \\
\text { health issues were } 44.1 \% \text {, family } \\
\text { relationship issues was } 19.6 \% \text {, } \\
\text { emotional issues was } 15.0 \% \text {, and } \\
\text { other issues was } 15.0 \%\end{array}$ \\
\hline
\end{tabular}


burnout experienced by health personnel caring for COVID-19 patients had an impact on their quality of life (QoL). Married healthcare professionals had significantly higher stress $(t=-2.380, \mathrm{p}=0.015, \mathrm{X}=61.9)$, trait anxiety $(t=3.012, \mathrm{p}=0.001, \mathrm{X}=44.21)$, and burnout $(t$ $=1.789, \mathrm{p}=0.002, \mathrm{X}=29.67)$ mean scores than single employees. In terms of professional standing, nurses had significantly greater mean burnout levels $(t=2.564, \mathrm{p}=$ $0.004, \mathrm{X}=8.91)$ than doctors $(\mathrm{X}=8.41)$ and other supporting personnel $(\mathrm{X}=8.14)$. Healthcare professionals with children had substantially higher average stress $(t=$ $1.876, \mathrm{p}=0.013, \mathrm{X}=67.78)$, anxiety $(t=3.356, \mathrm{p}=0.001$, $\mathrm{X}=9.97)$, and quality of life $(t=3.325, \mathrm{p}=0.001, \mathrm{X}=$ 49.11) ratings than those without children. ${ }^{27}$

Current study findings on the mental health of healthcare professionals during the COVID-19 epidemic have been found in Italy. Quality of life (QoL) and healthrelated Visual Analogue Scales, State-Trait Anxiety Inventory-Form Y1, Beck Depression Inventory, and post-traumatic stress disorder (PTSD) checklist for the Diagnostic and Statistical Manual of Mental Disorders (DSM-5) are the study tools. According to the study's findings, the health worker's mean \pm SD for life happiness and health concern was $6.8 \pm 2.2$ and $6.5 \pm 2.6$, respectively. According to the findings obtained, healthcare professionals who work in COVID-19 wards showed greater levels of depressive symptoms and post-traumatic stress symptoms (PTSS) than those who work in other healthcare units. $^{25}$

The current study on Psychological Symptoms was undertaken among Health Professionals in Spain following the first wave of the COVID-19 outbreak. Depression Anxiety Stress Scales (DASS-21) have been used to assess anxiety, stress, and depression, PTSD Checklist-civilian (PCL-C) was used to assess post-traumatic stress, and Professional Quality of Life (QoL) was used to assess compassion fatigue are the study instruments. According to the survey, 973 health professionals experienced greater workplace pressure during the pandemic period, as well as post-traumatic stress and compassion fatigue. The greatest perceived degree of post-traumatic stress and professional quality-of-life symptoms suffered by health professionals (low, medium, and high) was 72 (7.4\%). Among health professionals, the detected extremely severe degree of depression [47 (4.8\%)], anxiety [115 (11.8\%)], and stress symptoms [59 (6.1\%)] were observed. ${ }^{6}$

During the COVID-19 pandemic, new study findings on mental health outcomes and professional quality of life
(QoL) were documented among 893 respondents using a web-based, descriptive quantitative cross-sectional methodology. The study's instruments were feelings-related questionnaires, the Impact of Event Scale-Revised, the Connor-Davidson Resilience Scale (CD-RISC), and the Professional Quality of Life Scale (ProQoL). According to the study findings, female nurses and doctors working in the emergency unit had a higher proportion of psychological discomfort. The identified professional quality-of-life symptoms among workers in the COVID-19 special departments were satisfaction (40.12 \pm 4.3$)$, burnout $(32.30 \pm 7.7)$, and stress $(28.49 \pm 8.1) .^{23}$

During the COVID-19 epidemic in Guangdong, China, a cross-sectional survey of the study findings on the psychological health state of healthcare personnel was observed. The instruments used in the study include the QoL scale, the Zung Self-Rating Anxiety Scale (SAS), and the Zung Self-Rating Depression Scale (SDS). It was revealed that $221(24.34 \%)$ of the respondents had varying levels of anxiety, 299 (32.93\%) of them had depression, and $25.88 \%$ and $41.08 \%$ of the HCW respondents were concerned about themselves or their family members becoming infected with COVID-19. HCW had considerably greater morbidity of both anxiety and depression when compared to the control group. And, 221 (24.34\%) of the HCW participants were anxious, with a mean SAS score of 42.9, and 299 (32.93\%) were depressive. The mean SDS was 47.8. ${ }^{12}$

During the peak of the COVID-19 outbreak in Vietnam, cross-sectional research was conducted among front-line health professionals on outcomes of mental health and health-related quality-of-life, with 173 study respondents. According to the study findings, 20.2\% experienced depressive symptoms, $33.5 \%$ reported anxiety symptoms, and $12.7 \%$ expressed stress. Most issues were mentioned in the anxiety/depression component. The European Quality of Life-Five Dimension-Five Level Scale was used to assess health-related quality of life (HRQoL) (EQ-5D-5L). The EQ-5D-5L is a prominent HRQoL instrument that consists of five questions with a five-point Likert scale response. The EQ-5D-5L measured five aspects of HRQoL: mobility, self-care, usual activities, pain or discomfort, and mental health state. The median EQ-5D-5L index score was 0.93 (range: 0.27$1.00 ; 22.0 \%$ had perfect HRQoL scores), which was substantially higher among non-designated hospital HCWs (0.93 vs 0.87$).^{20}$ 
A recent study in Spain used a cross-sectional observational survey of 537 health professionals to report on primary and hospital care before COVID-19 among health workers in terms of professional quality of life and perceived stress. The Professional Quality of Life Scale (ProQoL) is a questionnaire with three subscales: Compassion Fatigue, Compassion Satisfaction, and Burnout Perceived Stress Scale (PSS-14) are the valid research tools applied in the study. This study assessed the professional quality of life (QoL) and perceived stress (PSS-14). According to the study findings, Professional quality of life and perceived stress in-hospital care related to, the greatest compassion satisfaction was 125 (42.5\%), compassion fatigue was 189 (64.3\%), and burnout was 108 $(36.7 \%){ }^{28}$

A web-based study report discovered health care providers treating patients with coronavirus disease-19 on quality of sleep and health-related quality of life using a cross-sectional design among 201 health care professionals. The Pittsburgh Sleep Quality Index, the Zung Self-rating Depression Scale, the 36-item Health Survey of the Medical Outcomes Investigation Short Form (SF36), and the 7-item Generalized Anxiety Disorder (GAD-7) Scale were used to assess QoL in this study (PSQI). The self-assessment of the present mental state demonstrated that $18.5 \%$ of medical workers who worked with COVID-19-positive patients described their mental status as excellent, $24.5 \%$ as very good, $42.3 \%$ as good, and $14.7 \%$ as poor. In comparison to pre-pandemic mental conditions, $64.3 \%$ of the patients reported deteriorating, and $61.5 \%$ said that the pandemic outbreak was impacting their mental health. ${ }^{22}$

During the COVID-19 epidemic, research on mental health and quality of life was undertaken among healthcare personnel in India. The study instruments include the Patient Health Questionnaire (PHQ-9), the Generalized Anxiety Disorder (GAD-7) questionnaire, the Quality of Life (QoL-1) visual analog scale, and multiple-choice questions with 12 options for assessment of the stressors. According to the study findings, a significant number of the sample $(92,47 \%)$ experienced symptoms of depression, anxiety $(98,50 \%)$, and low quality of life $(89,45 \%)$. According to the study's findings, during the COVID-19 epidemic, Indian health care workers had a significant incidence of depression and anxiety symptoms, as well as a negative quality of life. The study's low quality of life reported $(\mathrm{n}=89)$ was $45 \%$, with moderate-to-severe depression being $32(73 \%)$, moderate-to-severe anxiety being $39(\%)$, and moderate-to-severe depression and anxiety being $24(73 \%){ }^{29}$

A study was conducted in Australia on the mental health status of healthcare professionals vs other essential workers during the COVID-19 pandemic, with study participants including health care workers $(n=905)$, other essential workers $(\mathrm{n}=810)$, and the general population $(n=3443)$. The valid used study instruments are Depression Anxiety Stress Scales (DASS-21), Positive and Negative Affect Schedule (PANAS), and European Health Interview Surveys - Quality of Life (EUROHISQoL). The study findings included emotional experiences and quality of life-related to COVID-19 by the group with the estimated marginal mean standard error of healthcare worker (HCW; n=879-905) and Quality of life (EUROHIS-QoL, $\alpha=0.869$ ) 777.1 \pm 17.9 . EUROHIS-QoL stands for European Health Interview Surveys - Quality of Life (eight-item measure assessing the quality of life, rated on five-point Likert scales ranging from 1 to 5, with higher scores indicating greater levels of satisfaction). ${ }^{10}$

A cross-sectional research study reported in Romania and Bulgaria among community pharmacists during COVID-19 on the impact of COVID-19 on health-related quality of life and the perception of being vaccinated to prevent COVID-19, involving 395 respondents. In the study, the 15D instrument was utilized to evaluate the quality of life. The findings indicated the disparities in the quality of life of Romanian and Bulgarian pharmacists. The observed differences in sleeping, usual activities, mental function, discomfort and symptoms, depression, distress, and overall 15D score, with low values for distress, showed statistical significance. The findings of the health-related quality of life (HRQoL) with the total 15D score were $0.956 \pm 0.051$ in Romania $(\mathrm{n}=241)$ and $0.936 \pm$ 0.063 in Bulgaria $(\mathrm{n}=154) .{ }^{14}$

A study published in Romania among gastroenterologists on the impact of the COVID-19 pandemic on healthrelated quality of life, anxiety, and training among 96 respondents. The 15D (for evaluating health-related quality of life) and Endler multidimensional anxiety scales (EMAS - for assessing anxiety) are the validated instruments. According to the study's findings, the identified HRQoL among the Gastroenterology fellow group ( $\mathrm{n}=$ 64) was $0.966(0.055)$, and the Young specialist group $(\mathrm{n}=$ 32) was $0.966(0.055) \cdot(0.036) .^{30}$

A recent research study published from eight cities in Iran on the mental health and quality of life of obstetrics healthcare workers during a COVID-19 pandemic: a 
multicenter study with 599 study participants. The Patient Health Questionnaire-9 (PHQ-9), Multidimensional Scale of Perceived Social Support (MSPSS), and Short Form-36 (SF-36) surveys were used to assess Quality of Life (QoL) in this study. This survey has 36 items that are used to assess the state of the two major components of physical and mental health. The comparison of depression perceived social support, and quality of life in participants by contact status with red zone contacts with the Quality of life under Physical aspect components of Physical functioning was 90 [80-100], limitations due to physical health were 50 [25-100], the pain was 80 [55-100], and general health was 75 [55-90]. ${ }^{16}$

A study conducted in China among mental health professionals during the COVID-19 epidemic on workplace violence and its relationship with quality of life included 10,516 participants. The study instruments are, the Chinese version of the 9-item workplace violence scale (GAD-7) was used to assess participants' experiences with WPV, the 7-item Generalized Anxiety Disorder Chinese version (GAD-7) was used to evaluate the severity of anxiety symptoms, and QOL was evaluated using the first two items on the overall QOL derived from the World Health Organization Quality of Life Questionnaire - Brief Version (WHOQOL-BREF). According to the study findings, 1658 (15.8\%) of individuals who experienced workplace violence (WPV) $(n=1948)$ reported verbal abuse and/or threats, while 878 (8.4\%) indicated physical assault. The overall Quality of Life (QoL) of mental health professionals was $6.6(1.6 \%)$, whereas the non-WPV group $(\mathrm{N}=8568)$ QoL was $6.8(1.5 \%)$, and the WPV group $(\mathrm{N}=1948)$ QoL was 5.9. $(1.5 \%){ }^{31}$

A mid-pandemic nationwide survey of health care professionals' mental health and quality of life, with around 1685 participants, was reported during COVID-19. The valid study tools are the Patient Health Questionnaire-9, the General Anxiety Disorder-7, the Primary Care Posttraumatic Stress Disorder Screen, and the Alcohol Use Disorders Identification Test-C. The findings were, out of 1685 participants, 31\% (404 of 1311) confirmed mild anxiety, and 33\% (427 of 1311) clinically meaningful anxiety; 33\% (393 of 1341) reported mild depressive symptoms, and 17\% (233 of 1341) moderate-to-severe depressive symptoms; 34\% (64 of 1326) confirmed suicidal ideation; and 14\% (184 of 1300) were found to be positive for post-traumatic stress disorder. ${ }^{21}$

A cross-sectional research study literature was found from Liaoning province, China, among residents on the impact of the COVID-19 pandemic on mental health and quality of life among 263 (106 males and 157 females) participants. The study instruments are the Impact of Event Scale (IES), indicators of negative mental health impacts, social and family support, and mental healthrelated lifestyle changes. The participants' mean age was $37.7 \pm 14.0$ years, and $74.9 \%$ had a high level of education. The participants' mean IES score was $13.6 \pm 7.7$, indicating a mild stressful impact. Only $7.6 \%$ of the participants obtained an IES score $\geq 26$. The majority of participants $(53.3 \%)$ did not feel helpless as a result of the epidemic. The epidemic, on the other hand, caused $52.1 \%$ to feel horrified and apprehensive. Furthermore, the majority of participants (57.8-77.9\%) reported increased support from friends and family members, increased shared emotions, and care for family members and others. ${ }^{32}$

A national survey research study was published from China among frontline psychiatric practitioners during the COVID-19 pandemic depression and its association with quality of life from $n=10,516$ participants. The Depression and QOL of the respondents were evaluated using the Patient Health Questionnaire nine items (PHQ-9) and the World Health Organization Quality of Life Questionnaire-brief version (WHOQOL-BREF), correspondingly. The overall (Quality of Life) QoL of the study participants was 6.64 (1.60\%), whereas the QoL of no depression participants $(\mathrm{N}=7517)$ was $7.12(1.42 \%)$, and for individuals with depressive symptoms $(\mathrm{N}=2999)$ was $5.46(1.39 \%){ }^{33}$

During the COVID-19 pandemic, research findings on sleep characteristics were highlighted with a specific focus among health professionals using a cross-sectional technique among 170 study participants (100 health workers and 70 non-health workers). The study's tools include the Epworth Sleepiness Scale (ESS), the Pittsburgh Sleep Quality Index (PSQI), the Insomnia Severity Index (ISI), and the 17-item Hamilton Rating Scale (HRS). The study's major outcomes were, that self-reported insomnia, nightmares, sleepwalking, and sleep terrors are more prevalent among health workers who have poor sleep quality. At p-value of 0.05, the observed Insomnia Severity Index (ISI) total score (mean \pm SD) among health professionals was $7.83 \pm 5.29 .^{19}$

During the COVID-19 outbreak, a research study was reported on the mental health state of healthcare personnel among 5018 eligible survey respondents. The study's tools include the Symptom Checklist-90 (SCL-90) scale and a sociodemographic questionnaire. Economic problems were the most seriously impacted 52\% during the epidemic, followed by interpersonal communication problems 
(46.6\%), mental health issues $(46.4 \%)$, learn or work (45.0\%), body health issues (44.1\%), family relationship issues (19.6\%), emotional issues (15.0\%), and other issues $(15.0 \%){ }^{9}$

A study with 1290 respondents was undertaken on the Quality of Life Prior and During the COVID-19 Pandemic: A Nationwide Cross-Sectional Study with Brazilian Dietitians. The used study instrument was WHO-QOL-BREF. The findings of the study are emphasized with data on quality of life (QoL) before SARSCOV-2 (3.83 \pm 0.59$)$ and during the epidemic (3.36 \pm 0.66$)$ were significantly different. It was concluded with, SARSCOV-2 pandemic has had a detrimental influence on the QoL of Brazilian dietitians because health professionals experience changes in their lives as a result of their work pressure. $^{18}$

\section{Conclusion}

COVID-19 is an emerging disease that has been spreading among different countries with different variants or by undergoing various viral mutations since the end of 2019 and had a severe impact globally by distracting the physical, mental, and social wellbeing of individuals, particularly deviations in QoL of health professionals, by increasing work pressure and irrational fear of easily spreading of virus by direct contact with infected people during the delivery of emergency medical services to virus-affected people. As the predominant role of health professionals, meeting the requirements of sick people during pandemic situations, delivering effective health care services to hospitalized individuals by implementing comprehensive vital care to the needy individuals, and by handling emergency life-threatening conditions by standing on the frontlines while saving people's lives. The current analysis attempted to focus on the assessment of QoL led by health professionals during the COVID-19 pandemic crisis. Because health professionals are good at maintaining their health in all areas such as physical, mental, and social wellness, they may be more committed professionally at any pivotal times and can stand in the frontline by facing any professional challenges in providing care to ill people. All stakeholders in public and private health organizations must consider their primary obligation to health professionals to sustain their high quality of life. Various reviews have revealed their study findings on the quality of life of health workers during key stages of the COVID-19 epidemic. The majority of the findings indicate that there is a greater impact on the QoL of health professions due to various categories of issues such as disturbances in physical and mental health conditions during pandemics such as depression, anxiety, fear of viral transmission while providing care to hospitalized patients, family concerns burdens related to the uncertainty of the health conditions of family members, and reduced social interactions both personally and professionally. All of these problems, both directly and indirectly, are lowering the QoL of health professionals throughout the world. There is a need to analyze the personal and professional requirements of health professionals.

Personally aid health care workers by offering immediate health treatment to their family members and providing financial assistance to their family members if an emergency need persists. Professionally assist the health professional by supporting or increasing manpower, particularly by recruiting health care providers, to reduce or minimize the burden among the health professional, and by providing adequate personal protective equipment (PPE) kits while treating or being exposed to infected cases. Allowing them to address their needs and demands during working conditions, minimizing work pressures, controlling work-related violence and abusing behaviors in the workplace, understanding pandemic burdens among health professionals, and highly motivating the health professionals and recognizing the responsibilities were taken by the health professions while delivering care to the sick people or while delivering comprehensive care to all health care settings.

\section{Author Contributions}

All authors made a significant contribution to the work reported, whether that is in the conception, study design, execution, acquisition of data, analysis and interpretation, or in all these areas; took part in drafting, revising or critically reviewing the article; gave final approval of the version to be published; have agreed on the journal to which the article has been submitted; and agree to be accountable for all aspects of the work.

\section{Disclosure}

The author reports no conflicts of interest in this work.

\section{References}

1. Buselli R, Corsi M, Baldanzi S, et al. Professional quality of life and mental health outcomes among health care workers exposed to SarsCov-2 (Covid-19). Int J Environ Res Public Health. 2020;17(17):6180. doi:10.3390/ijerph17176180

2. Hadning I, Ainii NQ. An analysis of health workers' quality of life in Indonesia during COVID-19 pandemic. In: 4th International Conference on Sustainable Innovation 2020-Health Science and Nursing (Icosihsn 2020). Yogyakarta, Indonesia: Atlantis Press; 2021. doi:10.2991/ahsr.k.210115.085 
3. Abdelghani M, Mahdy R, El-Gohari H. Health anxiety to COVID-19 virus infection and its relationship to quality of life in a sample of health care workers in Egypt: a cross-sectional study. Arch Psychiatry Psychother. 2021;23(1):19-28. doi:10.12740/APP/130304

4. WHO. WHO Coronavirus (COVID-19) dashboard. Available from: https://covid19.who.int. Accessed December 16, 2021.

5. Zhu N, Zhang D, Wang W, et al. A novel Coronavirus from patients with Pneumonia in China, 2019. N Engl J Med. 2020;382(8):727733. doi:10.1056/NEJMoa2001017

6. Dosil M, Ozamiz-Etxebarria N, Redondo I, Picaza M, Jaureguizar J. Psychological symptoms in health professionals in Spain after the first wave of the COVID-19 pandemic. Front Psychol. 2020;11. doi:10.3389/fpsyg.2020.606121

7. Khan AG, Kamruzzaman M, Rahman MN, Mahmood M, Uddin MA. Quality of life in the COVID-19 outbreak: influence of psychological distress, government strategies, social distancing, and emotional recovery. Heliyon. 2021;7(3):e06407. doi:10.1016/j.heliyon.2021.e06407

8. Pappa S, Ntella V, Giannakas T, Giannakoulis VG, Papoutsi E, Katsaounou P. Prevalence of depression, anxiety, and insomnia among healthcare workers during the COVID-19 pandemic: a systematic review and meta-analysis. Brain Behav Immun. 2020;88:901907. doi:10.1016/j.bbi.2020.05.026

9. Liu Z, Wu J, Shi X, et al. Mental health status of healthcare workers in China for COVID-19 epidemic. Ann Glob Health. 2021;86(1):128. doi: $10.5334 / \operatorname{aogh} .3005$

10. Toh WL, Meyer D, Phillipou A, et al. Mental health status of healthcare versus other essential workers in Australia amidst the COVID-19 pandemic: initial results from the collate project. Psychiatry Res. 2021;298:113822. doi:10.1016/j.psychres.2021.113822

11. Deng D, Naslund JA. Psychological impact of COVID-19 pandemic on frontline health workers in low- and middle-income countries. Harv Public Health Rev Camb Mass. 2020;28. Available from https://www.ncbi.nlm.nih.gov/pmc/articles/PMC7785092/.

12. $\mathrm{Li} \mathrm{Q}$, Chen J, Xu G, et al. The psychological health status of healthcare workers during the COVID-19 outbreak: a cross-sectional survey study in Guangdong, China. Front Public Health. 2020;8. doi:10.3389/fpubh.2020.562885/full

13. Trumello C, Bramanti SM, Ballarotto G, et al. Psychological adjustment of healthcare workers in Italy during the COVID-19 pandemic: differences in stress, anxiety, depression, burnout, secondary trauma, and compassion satisfaction between frontline and non-frontline professionals. Int $J$ Environ Res Public Health. 2020;17(22):8358. doi:10.3390/ijerph17228358

14. Turcu-Stiolica A, Bogdan M, Subtirelu M-S, et al. Influence of COVID-19 on health-related quality of life and the perception of being vaccinated to prevent COVID-19: an approach for community pharmacists from Romania and Bulgaria. J Clin Med. 2021;10 (4):864. doi:10.3390/jcm10040864

15. Orsini A, Corsi M, Santangelo A, et al. Challenges and management of neurological and psychiatric manifestations in SARS-CoV-2 (COVID19) patients. Neurol Sci. 2020;1-14. doi:10.1007/s10072-020-04544-w

16. Vafaei H, Roozmeh S, Hessami K, et al. Obstetrics healthcare providers' mental health and quality of life during COVID-19 pandemic: multicenter study from eight cities in Iran. Psychol Res Behav Manag. 2020;13:563. doi:10.2147/PRBM.S256780

17. Waszkiewicz N. Possible special needs for mental online support in female and male health care workers during the COVID-19. J Med Virol. 2020;93:174-175. doi:10.1002/jmv.26273

18. Adjafre da Costa Matos R, Coelho de Almeida Akutsu RC, Puppin Zandonadi R, Braz Assunção Botelho R. Quality of life prior and in the course of the COVID-19 pandemic: a nationwide cross-sectional study with Brazilian Dietitians. Int J Environ Res Public Health. 2021;18(5):2712. doi:10.3390/ijerph18052712
19. Herrero San Martin A, Parra Serrano J, Diaz Cambriles T, et al. Sleep characteristics in health workers exposed to the COVID-19 pandemic. Sleep Med. 2020;75:388-394. doi:10.1016/j.sleep.2020.08.013

20. Manh Than H, Minh Nong V, Trung Nguyen C, et al. Mental health and health-related quality-of-life outcomes among frontline health workers during the peak of COVID-19 outbreak in Vietnam: a cross-sectional study. Risk Manag Healthc Policy. 2020;13:29272936. doi:10.2147/RMHP.S280749

21. Young KP, Kolcz DL, O’Sullivan DM, Ferrand J, Fried J, Robinson $\mathrm{K}$. Health care workers' mental health and quality of life during COVID-19: results from a mid-pandemic, national survey. Psychiatr Serv. 2021;72(2):122-128. doi:10.1176/appi.ps.202000424

22. Stojanov J, Malobabic M, Stanojevic G, Stevic M, Milosevic V, Stojanov A. Quality of sleep and health-related quality of life among health care professionals treating patients with coronavirus disease-19. Int J Soc Psychiatry. 2021;67(2):175. doi:10.1177/ 0020764020942800

23. Tomar BS, Suman S, Singh P, Raj P, Nathiya D. Mental health outcome and professional quality of life among healthcare workers during COVID-19 pandemic: a frontline-COVID survey. Hamdan Med J. 2020;13(4):196. doi:10.4103/HMJ.HMJ_53_20

24. Rajkumar RP. COVID-19 and mental health: a review of the existing literature. Asian $J$ Psychiatr. 2020;52:102066. doi:10.1016/j. ajp.2020.102066

25. Di Tella M, Romeo A, Benfante A, Castelli L. Mental health of healthcare workers during the COVID-19 pandemic in Italy. $J$ Eval Clin Pract. 2020;26(6):1583-1587. doi:10.1111/jep.13444

26. Buselli R, Baldanzi S, Corsi M, et al. Psychological care of health workers during the COVID-19 Outbreak in Italy: preliminary report of an occupational health department (AOUP) responsible for monitoring hospital staff condition. Sustainability. 2020;12(12):5039. doi: $10.3390 /$ su12125039

27. Çelmeçe N, Menekay M. The effect of stress, anxiety and burnout levels of healthcare professionals caring for COVID-19 patients on their quality of life. Front Psychol. 2020;11:3329. doi:10.3389/ fpsyg.2020.597624

28. Ortega-Galán ÁM, Ruiz-Fernández MD, Lirola M-J, et al. Professional quality of life and perceived stress in health professionals before COVID-19 in Spain: primary and hospital care. Healthcare. Multidisciplinary Digital Publishing Institute; 2020:484. doi:10.3390/healthcare8040484

29. Suryavanshi N, Kadam A, Dhumal G, et al. Mental health and quality of life among healthcare professionals during the COVID-19 pandemic in India. Brain Behav. 2020;10(11):e01837. doi:10.1002/brb3.1837

30. Ungureanu BS, Vladut C, Bende F, et al. Impact of the COVID-19 pandemic on health-related quality of life, anxiety, and training among young gastroenterologists in Romania. Front Psychol. 2020;11(579177). doi:10.3389/fpsyg.2020.579177

31. Xie X-M, Zhao Y-J, An F-R, et al. Workplace violence and its association with quality of life among mental health professionals in China during the COVID-19 pandemic. J Psychiatr Res. 2021;135:289-293. doi:10.1016/j.jpsychires.2021.01.023

32. Zhang Y, Ma ZF. Impact of the COVID-19 pandemic on mental health and quality of life among local residents in Liaoning Province, China: a cross-sectional study. Int J Environ Res Public Health. 2020;17(7):2381. doi:10.3390/ijerph17072381

33. Zhang -H-H, Zhao Y-J, Wang C, et al. Depression and its relationship with quality of life in frontline psychiatric clinicians during the COVID-19 pandemic in China: a national survey. Int $J$ Biol Sci. 2021;17(3):683. doi:10.7150/ijbs.56037 


\section{Publish your work in this journal}

The Journal of Multidisciplinary Healthcare is an international, peerreviewed open-access journal that aims to represent and publish research in healthcare areas delivered by practitioners of different disciplines. This includes studies and reviews conducted by multidisciplinary teams as well as research which evaluates the results or conduct of such teams or healthcare processes in general. The journal

covers a very wide range of areas and welcomes submissions from practitioners at all levels, from all over the world. The manuscript management system is completely online and includes a very quick and fair peer-review system. Visit http://www.dovepress.com/testimonials. php to read real quotes from published authors. 